\title{
A Self-Equalizing Photo Detector
}

\author{
Behrooz Abiri $^{1}$, Firooz Aflatouni ${ }^{1,2}$, Ali Hajimiri ${ }^{1}$ \\ ${ }^{1}$ Department of Electrical Engineering, California Institute of Technology, 1200 E California Blvd, Pasadena, CA 91125, USA \\ ${ }^{2}$ Department of Electrical and Systems Engineering, University of Pennsylvania, 200 South 33 ${ }^{\text {rd }}$ St., Philadelphia, PA 19104, USA
} babiri@caltech.edu

\begin{abstract}
A self-equalizing photo-detector (SEPD) that mitigates the bandwidth limitations of electro-optical components of optical communication systems is demonstrated, enabling higher rate of data transmission, using slower components. Unlike other all-optical equalization schemes, SEPD is optically wide band, thus does not require wavelength tuning.
\end{abstract}

Keywords-feed-forward equalizer; equalization; silicon photonics; active optical cable; photo detector

\section{INTRODUCTION}

The implementation cost of optical communication channels has been an impediment to broad deployment of high speed optical systems for short range front-end of communication networks. The advances in silicon photonics enabling co-integration of electronics and photonics on the same substrate combined with the availability of low-cost laser light sources have enabled low-cost high-speed optical connectivity solutions [1].

Active optical cables have become the de-facto standard connectivity solutions in data centers and high performance computing clusters. The main bottleneck limiting the data rate in these cables is the bandwidth of the electro-optical components, such as the optical modulator and the photodiodes. Equalization techniques that have been widely used in copper based communication channels [2] can also be used for optical communication channels [3]. The work presented in this paper demonstrates a novel hybrid electro-optical equalization approach on an integrated silicon photonic chip, hence not constrained by the bandwidth and speed limitations of the following electronic circuitry and lowering the overall power consumption of the system. Also, unlike other optical equalizers that utilize a narrowband optical filter to shape the spectrum of modulated data (e.g., [4]), the proposed scheme does not have a strong wavelength dependency; therefore it can be used without the need of accurate optical wavelength tuning.

\section{DESIGN AND FABRICATION}

An Equalizer usually acts as a high-pass filter that cancels out channel attenuation at higher frequencies. The high-pass filter can be placed anywhere in the link, e.g. in the transmitter or receiver, or can be implemented through digital signal processing, as long as the link is substantially linear. In optical links, this high pass filter can be simply implemented as an electro-optical hybrid using the components available in a typical silicon photonics process. Such electro-optical equalizer can take advantage of the high optical bandwidth and hence

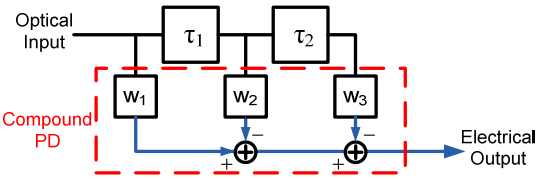

(a)

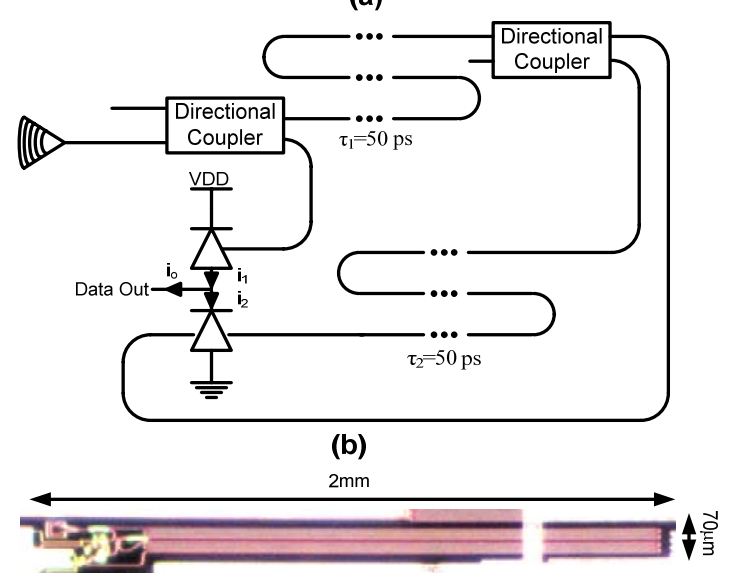

(c)

Figure 1. Proposed self-equalizing photo detector. (a) Conceptual block diagram, (b) detailed implemented block

diagram, and (c) the die photo of fabricated detector

provide better equalization than what can be achieved with a purely electronic solution. At the same, the electronic eliminates the strong wavelength dependence of the narrowband grating-based optical equalization solutions (e.g., [4]).

Figure 1 shows the block diagram and the die photo of the designed self-equalizing photo detector (SEPD). A laser source emitting in $1550 \mathrm{~nm}$ is coupled into the silicon photonic chip through a lensed grating coupler. An imbalanced evanescent mode coupler split the light into two branches guiding most of the input light to a SiGe photodiode, $\mathrm{PD}_{1}$. The remainder of the input light is guided to the second imbalanced coupler through a 50ps delay line.. A fraction of the delayed optical signal is incident on the second photo detector $\mathrm{PD}_{2}$ without any extra delay while the remainder of the optical signal is passed through a second 50ps delay line before being fed to $\mathrm{PD}_{2}$. The electrical connection between $\mathrm{PD}_{1}$ and $\mathrm{PD}_{2}$ is such that the photocurrent of $\mathrm{PD}_{2}$ is subtracted from the photocurrent of $\mathrm{PD}_{1}$, producing opposite polarities for $w_{1}$ with respect to $w_{2}$ and $w_{3}$. This architecture effectively forms a high pass filter that equalizes and detects the optical signal. Assuming optical delays of $\tau_{1}$ and $\tau_{2}$ and directional couplers with coupling ratios 
of $\alpha_{1}$ and $\alpha_{2}$, the frequency response of the detector can be calculated as

$$
\text { (1) } i_{o}=\frac{R P}{1-\alpha_{1}}\left[1-w_{1} e^{-2 i \pi f \tau_{1}}-w_{2} e^{-2 i \pi f\left(\tau_{1}+\tau_{2}\right)}\right]
$$

where $R$ is the responsivity of the photo detectors, $P$ is the optical power coupled into the chip, $w_{1}=\left(1-\alpha_{2}\right) \alpha_{1} / 1-\alpha_{1}$, and $w_{2}=\alpha_{1} \alpha_{2} / 1-\alpha_{1}$. As the equation suggests, the equalizer represents the frequency response of a finite impulse response (FIR) filter.

The silicon photonic chip was implemented in OpSIS/IME SOI process with silicon thickness of $220 \mathrm{~nm}$ on a $2 \mu \mathrm{m}$ buried oxide layer [1]. The light was coupled into the chip via a grating coupler and single mode on chip waveguides with group index of 4.2 were used as delay lines. The process also incorporates a Ge epitaxial layer that is used to create the photo-detectors.

\section{Measurement Results}

The effectiveness of the self-equalizing photo-detector (SEPD) is demonstrated in a $12.5 \mathrm{Gbps}$ data link and the performance of the proposed detector was compared to a standalone $35 \mathrm{GHz}$ photo diode. The frequency response of the electro-optical test channel is shown in Figure 2. This response includes the bandwidth limitation of the optical modulator, its driver, and the electrical cables that connect the pattern generator to the modulator and the photo-detector to the oscilloscope. The channel has around $12.5 \mathrm{~dB}$ of attenuation at the Nyquist frequency resulting in a closed eye diagram when measured with the 35GHz photo-detector (Figure 3(a)).

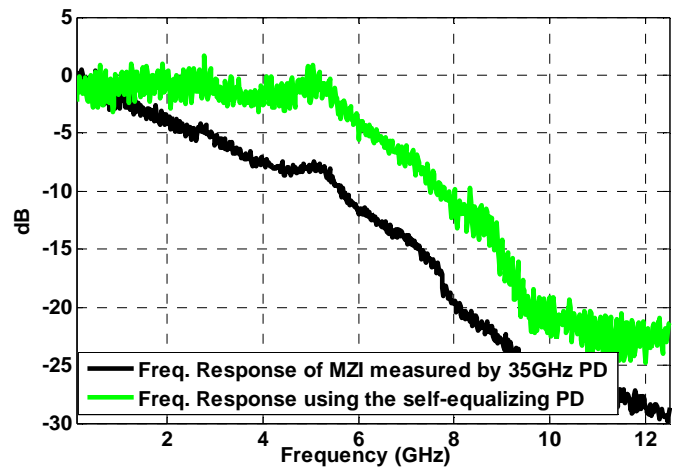

Figure 2. Electro-optical frequency response of the channel with a 35GHz PD and with proposed self-equalizing PD. The

self-equalizing $\mathrm{PD}$ provides up to $7 \mathrm{~dB}$ of equalization.

Figure 3(b) shows that resulting eye diagram of using the proposed self-equalizing photo-detector in place of the $35 \mathrm{GHz}$ $\mathrm{PD}$. The open eye diagram shows that the proposed detector is able to equalize the optical signal successfully.

\section{CONCLUSION}

A self-equalizing photo-detector (SEPD) capable of equalizing an electro-optical channel with $12.5 \mathrm{~dB}$ of attenuation is presented. The proposed detector uses low loss optical waveguides to achieve true time delay that is required for creating a wavelength independent feed-forward equalizer. The proposed photo-detector hence enables higher rate of data transfer in bandwidth limited optical communication systems.

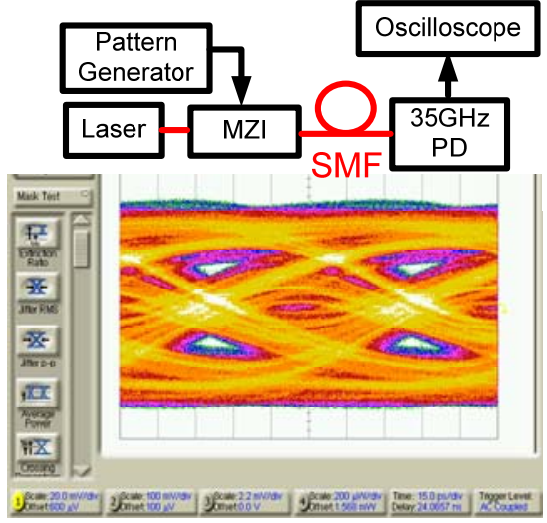

(a)

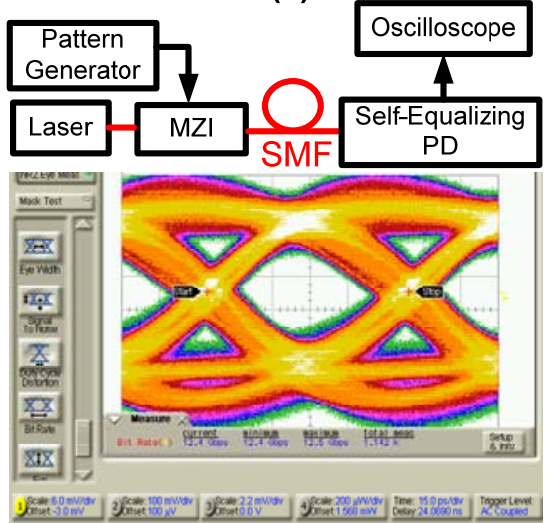

(b)

Figure 3. (a) Eye diagram of the electro-optical channel measured using a $35 \mathrm{GHz}$ photo diode. (b) The eye diagram obtained using the proposed self-equalizing photo-detector

\section{ACKNOWLEDGMENT}

The authors would like to thank OpSIS for chip fabrication, Prof. Tom Baehr-Jones, and Prof. Michael Hochberg for their help in chip design verification.

\section{REFERENCES}

[1] M. Streshinsky, R. Ding, Y. Liu, A. Novack, C. Galland, A. Lim, P. Guo-Qiang Lo, T. Baehr-Jones, and M. Hochberg, "The Road to Affordable, Large-Scale Silicon Photonics," Opt. Photon. News 24(9), 32-39 (2013).

[2] S. Gondi, and B. Razavi, "Equalization and Clock and Data Recovery Techniques for 10-Gb/s CMOS Serial-Link Receivers," IEEE J. of Solid-State Circuits, vol. 42, no. 9, pp. 1999-2011, Sept. 2007.

[3] I. Lu, J. Shi, H. Chen, C. Wei, S. Tsai, D. Hsu, Z. Wei, J. Wun, and J. Chen, "Ultra Low Power VCSEL for 35-Gbps 500-m OM4 MMF Transmissions Employing FFE/DFE Equalization for Optical Interconnects," in Optical Fiber Communication Conference 2013, OSA Technical Digest, paper JTh2A.75.

[4] Zhao Xiaoxue, K. Hasebe, T. Sakaguchi, F. Koyama, C.J. ChangHasnain, N. Nishiyama, C. Caneau, Zah Chung-en, "Tunable Optical Equalizer Using Diffraction Grating Filters," IEEE Photonics Technology Letters, vol.20, no.18, pp.1590,1592, Sept.15, 2008. 